\title{
1 Global environmental drivers of marine fish egg size
}

2 Diego R. Barneche ${ }^{1, \S, *}$, Scott C. Burgess ${ }^{2}$, Dustin J. Marshall ${ }^{1}$

$3{ }^{1}$ Centre for Geometric Biology / School of Biological Sciences, Monash University, Clayton Vic 3800, Australia

$4 \quad{ }^{2}$ Department of Biological Science, Florida State University, Tallahassee, FL, 32306, USA

5 \$Present address: School of Life and Environmental Sciences, The University of Sydney, Sydney NSW 2006,

6 Australia

7 Running title: Biogeography of marine fish egg size

8 Keywords: Geometric Biology, predictability, life history, trait biogeography, maternal effects,

9 ocean warming

10 Type of article: Research paper

11 Word Count: 4,448 (Main text); 297 (Abstract)

12 Number of references: 51

13 Number of figures: 2

14 Correspondence should be sent to: Diego R. Barneche, Centre for Geometric Biology /

15 School of Biological Sciences, Monash University, Clayton, Vic. 3800, Australia (Tel: +61

16 (3) 9905 5100; Email: barnechedr@gmail.com). 


\section{Abstract}

18 Aim: To test long standing theory on the role of environmental conditions (both mean and

19 predictability) in shaping global patterns in the egg sizes of marine fishes.

20 Location: Global $\left(50^{\circ} \mathrm{S}\right.$ to $\left.50^{\circ} \mathrm{N}\right)$.

21 Time period: 1880 to 2015 .

22 Major taxa studied: Marine fish.

23 Methods: We compiled the largest geo-located dataset of marine fish egg size (diameter) to

24 date ( $n=1,078$ observations; 192 studies; 288 species; 242 localities). We decomposed sea

25 surface temperature (SST) and chlorophyll-a time-series into mean and predictability (sea-

26 sonality and colour of environmental noise - i.e. how predictable the environment is between

27 consecutive time steps), and used these as predictors of egg size in a Bayesian phylogenetic

28 hierarchical model. We test four specific hypotheses based on the classic discussion by Rass

29 (1941), as well as contemporary life-history theory, and the conceptual model of Winemiller

30 \& Rose (1992).

31 Results: Both environmental mean and predictability correlated with egg size. Our parsimo-

32 nious model indicated that egg size decreases by c. 2.0 -fold moving from $1^{\circ} \mathrm{C}$ to $30^{\circ} \mathrm{C}$. En-

33 vironments that were more seasonal with respect to temperature were associated with larger

34 eggs. Increasing mean chlorophyll-a, from 0.1 to $1 \mathrm{mg} \mathrm{m}^{-3}$, was associated with a c. 1.3 -fold

35 decrease in egg size. Lower chlorophyll-a seasonality and reddened noise were also associ-

36 ated with larger egg sizes - aseasonal but more temporally autocorrelated resource regimes

37 favoured larger eggs.

38 Main conclusions: Our findings support results from Rass (1941) and some predictions from

39 Winemiller \& Rose (1992). The effects of environmental means and predictability on marine

40 fish egg size are largely consistent with those observed in marine invertebrates with feeding

41 larvae, suggesting important commonalities in how ectotherm egg size responds to environ-

42 mental change. Our results further suggest that anthropogenically-mediated changes in the

43 environment will have profound effects on the distribution of marine life histories. 
45 Life-history traits vary systematically along environmental gradients, particularly with latitude. For example, latitude covaries with adult body size (e.g. mammals, birds, fish,

47 insects; Bergamnn's rule; Meiri, 2011), offspring size (Rass's rule; Rass, 1941; Marshall, 48 1953; Marshall et al., 2012), developmental mode (Thorson's rule; Thorson, 1950; Marshall 49 et al., 2012), and dispersal capacity (O'Connor et al., 2007). For years, biogeographers 50 and life-history theoreticians have assumed that the latitudinal gradients in life history are 51 driven by differences in average conditions (e.g. mean temperature), but means are not the 52 only moments of the distribution that vary in space and influence life-history evolution (e.g. 53 Vasseur et al., 2014; Marshall \& Burgess, 2015).

Although ecologists have traditionally emphasised the role of environmental means across

55 latitudes as drivers of latitudinal variation, classic biogeography treatises recognised that other 56 components of environmental factors also vary systematically with latitude, particularly en57 vironmental predictability (Cohen, 1966; Slobodkin \& Sanders, 1969; Brown, 1973; Crump, 58 1981). Environments can be more or less predictable in multiple ways. First, environments 59 can be predictable if they remain relatively constant over time - temperature in much of the 60 deep sea is a good example of such conditions. Second, environments can be predictable if 61 they change in very regular ways - for example, highly seasonal environments, while vari62 able, are also predictable in the timing of events - temperate latitudes are likely to be warmer 63 and drier in summer relative to winter for example. Finally, environments can also be pre64 dictable if conditions at one time are strongly correlated with conditions at some point into the 65 future - i.e. there is a strong temporal autocorrelation in local conditions. For example, if an 66 environment is highly autocorrelated, then conditions now are a good indicators of conditions 67 later. This type of predictability occurs when the colour of environmental noise is reddened 68 (Vasseur \& Yodzis, 2004; Burgess \& Marshall, 2014). Importantly, all of these types of pre69 dictability (constancy, seasonality and autocorrelation) can differ across latitudes, and each 70 type of predictability will affect life-history evolution differently (Travis, 2001; Burgess \&

71 Marshall, 2014; Marshall \& Burgess, 2015; Rubio de Casas et al., 2017). 
and above the effects of environmental means (Parker \& Begon, 1986; Travis, 2001). For example, theory predicts that fecundity will be optimised by producing many small eggs in environments with predictable food regimes (Smith \& Fretwell, 1974; Parker \& Begon, 1986; Rollinson et al., 2013). Moreover, if environmental differences across space are relatively stable and predictable over time, theory predicts, and evidence suggests, that dispersal will not be favoured (Hastings, 1983; Fronhofer et al., 2014). On the other hand, mothers in unpredictable environments should produce fewer and larger offspring (i.e. a conservative bethedging approach Einum \& Fleming, 2004). Contradictions are common however, for example, where populations inhabiting increasingly harsh streams produced more numerous and smaller eggs (Morrongiello et al., 2012). Initial studies of trait biogeography support these theoretical predictions - latitudinal gradients in marine invertebrate life histories are driven strongly by changes in predictability of both food and temperature from the tropics to the poles (Marshall \& Burgess, 2015). But overall, biogeographical studies of how environmental predictability shapes life-history variation at large spatial scales remain exceedingly rare (Marshall \& Burgess, 2015; Rubio de Casas et al., 2017).

In fishes, Rass (1941) and Marshall (1953) first noted that lower latitudes and warmer temperatures are often associated with smaller egg sizes. Winemiller \& Rose (1992) proposed a conceptual argument that life histories encompass a continuum space among three major strategies, and that the interactions among juvenile survival, adult fecundity, and maturity are shaped not only by the average environmental state, but also by its predictability (see hypotheses below). Twenty-five years later, formal tests on how (and if) environmental mean and predictability consistently affect marine fish eggs over broad spatial scales are still lacking, despite a rich theoretical body of literature (Cohen, 1966; Smith \& Fretwell, 1974; Parker \& Begon, 1986; Winemiller \& Rose, 1992; Travis, 2001; Einum \& Fleming, 2004).

Species-level spawning mode is also known to correlate with egg size. For instance, species that exhibit parental care (i.e. brooders) are known to have larger eggs when compared to many pelagic- and demersal-spawning species (Winemiller \& Rose, 1992). Also, demersal spawners have on average larger eggs than pelagic spawners, followed by scatterer spawners (i.e. species who deposit their eggs on the benthos, but have a long pelagic stage when compare to ordinary demersal spawners) (Kasimatis \& Riginos, 2016). These correlations 
between spawning mode and egg size might exhibit a strong phylogenetic effect (e.g. most Tetraodontiformes are scatterers, Syngnathidae and Apogonidae are brooders; Kasimatis \& Riginos, 2016). However, it is unknown whether the effects of spawning mode remain substantial after accounting for the effects of environmental variables, and phylogeny on egg size.

Here we present a formal analysis on the role of the environment in shaping marine fish egg size. In doing so, we first compile the most comprehensive dataset of marine fish egg sizes which dates back to 1880 , and use it to explicitly test the following hypotheses: egg size responds to changes in average temperature $(H 1)$, productivity $(H 2)$, some form of environmental predictability $(H 3)$, and spawning mode $(H 4)$. Particularly, within $H 1$, we predict that egg size will decrease with increasing temperature. Within $H 2$, we predict that egg size will decrease with increasing average food regimes. For $\mathrm{H3}$, we derive predictions inspired by the conceptual model of Winemiller \& Rose (1992): high seasonality and low average food regimes drive declines in egg size (Periodic strategy); white-noised environments (i.e. unpredictable) with low seasonality drive declines in egg size (Opportunistic strategy); predictable environments with low average food regime drive egg size to increase (Equilibrium strategy). For $H 4$, we predict that after correcting for phylogeny, spawning mode will not significantly affect egg size. We apply this hypothesis-testing approach using Bayesian phylogenetic hierarchical models in order to account for shared evolutionary history among species, as well as independent evolutionary changes unique to each species.

\section{Materials and Methods}

\section{Egg size data compilation}

We began by compiling data from multiple previous compilations that provided references on egg size (diameter in $\mathrm{mm}$ ) for different species of marine fishes (Fritzsche, 1978; Duarte \& Alcaraz, 1989; Elgar, 1990; Moser, 1996; Einum \& Fleming, 2002; Hixon et al., 2014; Kasimatis \& Riginos, 2016). We used only raw data from original references (data extracted from tables or directly from figures using DataThief software). We also investigated papers cited in these original references, repeating this process exhaustively. Importantly, because 
131 we are primarily interested in the role of the environment, we only collected data from pub-

132 lications that reported the origin of specimen collection for populations directly collected

133 from the wild or that were transferred from the wild to an aquarium facility immediately be-

134 fore the measurements. Eggs that were measured immediately following fertilisation (i.e. no

135 change in size) were also included. For eggs that present ellipsoidal, elongated shapes, we

136 used both diameters (long, $l$, and short, $s)$ to first calculate the volume, $V\left(\mathrm{~mm}^{3} ; V=(4 / 3) \times\right.$

$\left.137 p i \times(l / 2) \times(s / 2)^{2}\right)$, and then back transformed to diameter of sphere with equivalent volume

$138\left(\left[((V \times 3) /(4 \times p i))^{(1 / 3)}\right] \times 2\right)$. Some papers reported mean egg size for each female while oth-

139 ers reported means across multiple females, so we are not able to properly consider the coef-

140 ficient of variation in egg size at the same scale across all data (see Einum \& Fleming, 2002).

141 Our dataset includes 1,078 observations from 192 studies between 1880 to 2015, and includes

142288 species.

\section{Environmental predictability and data}

144 We here consider two components of environmental predictability (Colwell, 1974; Marshall

145 \& Burgess, 2015; cf. Rubio de Casas et al., 2017). The first component is seasonality, which

146 entails the regularity in the timing and magnitude of fluctuations in the average environmental

147 state over seasons; it is expected to influence the evolution of egg size (Winemiller \& Rose,

148 1992). The second type is the colour of environmental noise (Halley \& Kunin, 1999; Vasseur

$149 \&$ Yodzis, 2004), which is defined by how predictable and similar the environment is between

150 successive time points, or how far into the future the environmental state is likely to stay the

151 same, independent of the mean environmental state (Marshall \& Burgess, 2015).

We wrote an R package (noaaErddap, available on www.github.com/dbarneche/noaaErddap) to download time-series data from NOAA on chlorophyll-a $\left(\mathrm{mg} \mathrm{m}^{-3} ; 0.08^{\circ}\right.$ resolution), which is used as proxy for environmental food regime, and sea surface temperature (hereafter SST; ${ }^{\circ} \mathrm{C} ; 0.25^{\circ}$ resolution), covering a 10 -year period from 1997 to 2007 . Chlorophyll-a data were recorded every eight days ( $\Delta t=8$ days, unevenly distributed) and SST data were recorded every day ( $\Delta t=1$ day, evenly distributed). For both SST and chlorophyll-a, we extracted the median environmental value within a $200-\mathrm{km}$ radius around each coordinate in our dataset. Following the approach adopted in Marshall \& Burgess (2015), only coordinates that had < 
$13 \%$ of the time series missing were used, hence all the data from coordinates above $50^{\circ}$ of latitude were excluded for the analysis (i.e. temporal coverage $<87 \%$ ).

Our calculations to decompose predictability into seasonality and colour of environmental noise are identical to those described by Marshall \& Burgess (2015). We first removed linear trends by extracting the residuals from a linear regression model fitted to the raw time series. For each coordinate, seasonality was estimated as the fraction of the total variance that is due to predictable seasonal periodicities, $a /(a+b)$, where $a$ is the variance of the seasonal trend, and $b$ is the variance of the residual time series (i.e. the time series after the seasonal trend was removed). The seasonal trend was estimated by binning the time-series data into monthly intervals, averaging each month across the 10 years, then re-creating a seasonal time-series data set on the same time scale of the original data using a linear interpolation between the monthly midpoints. The colour of environmental noise was estimated using the $1 / f^{\theta}$ family of noise models (Halley \& Kunin, 1999; Vasseur \& Yodzis, 2004), where white noise $(\theta=0)$ occurs when there is no correlation between one measurement and the next, while for reddened noise $(\theta>0)$, there is some correlation between measurements separated by a finite timescale. To do so, we first calculated a residual time series by subtracting the corresponding seasonal value from each data point in the time series. The spectral density (i.e. variance in the residual time series) was assumed to scale with frequency, $f$, according to an inverse power law, $1 / f^{\theta}$. The spectral exponent $\theta$ was estimated as the negative slope of the linear regression of $\log _{e}$ spectral density as a function of $\log _{e}$ frequency. Spectral density was estimated in R using the spectrum function form the stats R package v. 3.4.3 for the evenly-distributed SST time series, and the Lomb-Scargle function $l s p$ from the lomb R package v. 1.0 for the unevenlydistributed chlorophyll-a time series (Glynn et al., 2006). Spectral densities and subsequent $\theta$ 's were calculated between the frequencies of $2 / n \Delta t$ and $1 / 2 \Delta t$ ( $n=$ number of observations in the time series), which translates to periods of 16 days to 5.05 years for chlorophyll-a, and 2 days to 5.05 years for SST. Both seasonality and colour of environmental noise metrics are implemented in an R package (envPred, available on www.github.com/dbarneche/envPred). 
Phylogenetic relatedness might influence broad-scale variation in fish life-history traits (Kasimatis \& Riginos, 2016). From an evolutionary perspective, closely related species might have a higher likelihood of sharing some ancestral-state trait (Pagel, 1999), such as egg-type (pelagic, demersal, brooded). At the same time, species may present unique variations in traits that are independent of phylogenetic non-independence. From a technical perspective, both of these unmeasured biological factors (i.e. species uniqueness and non-independence), as well as the possibility for different species composition to underlie differences in egg size across latitudes, likely contribute to variance in a particular life-history trait (Hadfield \& Nakagawa, 2010) and, consequently, it is necessary to account for these possible effects. To do so, we created a tree from the Open Tree of Life (OTL) using the rotl R package v. 3.0.3 (Michonneau et al., 2016) in order to test for significant phylogenetic heritability in our models (Housworth et al., 2004). We first downloaded the full Actinopterygii tree from OTL ( $n=38,939$ tips) and then added species from our dataset that were missing in the tree: the Pomacentridae species relationship followed a recent consensus topology (Frédérich et al., 2013) and we inserted them as a sister group to the family Labridae; Heterostichus rostratus (family Clinidae), and Sphyraena argentea (family Sphyraenidae) were respectively inserted right next to the families Blenniidae and Carangidae (Betancur-R. et al., 2013). This tree was pruned to retain focal species only, and then used to derive a variance-covariance matrix based on Brownian evolution. The tree included a total of 30 polytomies. Branch lengths are unknown for the phylogeny, so the arbitrary method of Grafen (1989) was applied, whereby branch lengths are set to a length equal to the number of descendant tips minus one.

We compiled spawning mode for all 288 species in our dataset. We classified species according to 5 categories: Demersal: $n=188$ obs., 65 spp; Mouth brooder: $n=15$ obs., 13 spp; Pelagic: $n=836$ obs., 180 spp; Pouch brooder: $n=19$ obs., 15 spp; Scatterer: $n=20$ obs., $15 \mathrm{spp}$. Information on spawning modes were obtained from the literature (e.g. Kasimatis \& Riginos, 2016), www.FishBase.org, and by consulting with experts. We were able to reliably reference the spawning mode of 262 species (91\%). The information on spawning mode

5 for the remaining 26 species was approximated based on other species within the same family. Removing these species had no effect in our results (Table S1). The spawning mode in- 
217 formation and associated references can be directly downloaded from our GitHub repository

218 (https://github.com/dbarneche/fishEggSize/blob/master/data/spawningMode.csv).

\section{Testing hypotheses $\mathrm{H1}-\mathrm{H} 4$}

220 Before running our statistical analysis, we first checked for any systematic correlation among

221 our predictor variables (Table 1). Temperature mean and its predictability components were

222 strongly correlated (i.e. $\sim 0.7$; Dormann et al., 2013) and, given this caveat, we interpret our

223 temperature model coefficients as being non-independent. We note however that the colour of

224 temperature noise was not a significant variable and therefore dropped from our parsimonious

225 model (see Results section).

226 The full model was constructed using all the original six variables with the following struc-

227 ture:

$$
\ln E=\beta X+\gamma_{s p p}+\gamma_{p h y}+\varepsilon
$$

228 where $\ln E$ represents $\log$-transformed egg size, $\beta$ is a vector of 7 fixed-effect coefficients,

229 with 1 intercept and 6 slopes each corresponding to an environmental variable (i.e. both SST

230 and chlorophyll-a averages, seasonalities, and colours of environmental noise) in the model

231 matrix $X . \gamma_{s p p}$ and $\gamma_{p h y}$ are respectively vectors of random-effect coefficients that account for

232 residual intercept deviations attributable to species uniqueness and patterns of relatedness as

233 described by the phylogeny, and $\varepsilon$ is the model unexplained residual variation.

Fixed effects were assigned weakly informative priors following a Gaussian distribution.

235 Standard deviations $\left(\sigma_{\gamma_{s p p}}, \sigma_{\gamma_{p h y}}\right.$ and $\left.\sigma_{\varepsilon}\right)$ were assigned more informative priors following

236 a Student- $t$ distribution to speed-up model convergence (notice, though, that the full model

237 was first fitted with weakly informative priors in order to obtain estimates for the more in-

238 formative priors). We account for the phylogenetic non-independence among species follow-

239 ing the method of Hadfield \& Nakagawa (2010), where the non-independence random-effect

240 coefficients $\left(\gamma_{p h y}\right)$ are distributed following a multivariate normal distribution with means

241 of zero (such that $\gamma_{p h y}$ represents actual deviations from the model intercept) and a single

242 standard deviation $\left(\sigma_{\gamma_{p h y}}\right)$ which is weighted by the variance-covariance matrix, $A$, obtained 
243 from the tips of the phylogeny (i.e. $\sigma_{\gamma_{p h y}} \times A$, with $A$ being obtained using the $\mathrm{R}$ package 244 ape version 5.0; Paradis et al., 2004). The phylogenetic heritability (equivalent to Pagel's $245 \lambda$; Pagel, 1999; Hadfield \& Nakagawa, 2010), was estimated as the proportion of total vari246 ance, conditioned on the fixed effects, attributable to the random effect of phylogeny (i.e.

$\left.247 \sigma_{\gamma_{p h y}}^{2} /\left(\sigma_{\gamma_{s p p}}^{2}+\sigma_{\gamma_{p h y}}^{2}+\sigma_{\varepsilon}^{2}\right)\right)$. The posterior distributions of model parameters were estimated 248 using Markov chain Monte Carlo (MCMC) methods with NUTS sampler using the R pack249 age brms version 2.1.0 (Bürkner, 2017) by constructing three chains of 15,000 steps, including 2507,500 -step warm-up periods, so a total of 22,500 steps were retained to estimate posterior dis251 tributions (i.e. $(15,000-7,500) \times 3=22,500)$.

252 We performed a model selection procedure by first dropping any non-significant slope 253 (i.e. those whose $95 \%$ posterior credible intervals overlap zero) and then testing whether this 254 simpler model was significantly better than the original full model. After this procedure, we 255 also tested whether the best of the two models was significantly improved by adding egg 256 spawning mode. Model comparisons were done using leave-one-out cross-validation (LOO), 257 which, similarly to widely applicable information criterion (WAIC), is a fully Bayesian model 258 selection procedure for estimating pointwise out-of-sample prediction accuracy (Vehtari et $259 a l ., 2016)$. We calculated the expected $\log$ pointwise predictive density $\left(\widehat{e l p}_{l o o}\right)$ using the 260 log-likelihood evaluated at the posterior simulations of the parameter values (Vehtari et al., 261 2016). We calculated $p$-values for the pairwise differences in $\widehat{e l p d}$ loo $\left(\Delta \widehat{e l p d} d_{\text {loo }}\right)$ using stan262 dard errors (s.e.) and a normal probability density function. This method of calculating s.e.'s 263 is reliable for data sets with many observations ( $n=1,078$ in our analysis) because the dis264 tribution of $\Delta \widehat{e l p d}_{l o o}$ is well approximated by a Gaussian distribution (Vehtari et al., 2016). 265 Results were similar using $K$-fold cross-validation $(K=10)$. Model comparison was imple266 mented using the loo R package (Vehtari et al., 2016) version 1.1.0. All analyses, tables and 267 figures are fully reproducible in R. Data and code are available via the GitHub repository 268 (https://github.com/dbarneche/fishEggSize).

\section{Results}

270 Fish egg size varied in diameter from $0.24 \mathrm{~mm}$ to $6.5 \mathrm{~mm}$, spanning 1.4 orders of magnitude 271 in egg size variation. There was a significant relationship between latitude and egg size ( $p<$ 
0.0001), although only $3.5 \%$ of variation in egg size is explained by latitude alone (Fig. 1).

For every 10 degrees of latitude moving poleward, egg size increases by $7 \%$ on average.

Our parsimonious model contains all original variables except the colour of temperature noise; the full model had a lower average predictive accuracy over the parsimonious model $\left(\Delta \widehat{e l p d} d_{l o o}=-1.6 ; p=0.399\right)$. Although adding spawning mode to this model improved the average predictive accuracy $\left(\Delta \widehat{e l p d}_{l o o}=6.1\right)$, it was not significant $(p=0.136)$, and we therefore retained the simpler model as the parsimonious model (i.e. rejected hypothesis $H 4$; Table 2). After accounting for the fixed effects, the majority of the variance observed in the residuals is explained by substantial heritability among species (95.5\%; 95\% CI: 91.6\% - 97.7\%), suggesting a substantial effect of phylogeny, and a minor contribution of non-heritable variation within species $(2.4 \%$; $95 \%$ CI: $1.1 \%-5.0 \%)$.

In agreement with our prediction of hypothesis $H 1$, the parsimonious model indicated that egg size decreases systematically with increasing mean temperature (Fig. 2a). Particularly, the mean temperature slope $\left(\beta_{S S T}=-0.02 ; 95 \% \mathrm{CI}:-0.03-0.01\right)$ suggests that egg size will decrease by c. 2.0 -fold moving from $1{ }^{\circ} \mathrm{C}$ to $30^{\circ} \mathrm{C}$ after accounting for the effects of environmental predictability and mean food regime. Environments that were more seasonal with respect to temperature had lower mean temperatures, and were therefore associated with larger egg sizes $\left(\beta_{\text {SeasonSST }}=0.82 ; 95 \%\right.$ CI: $\left.0.47-1.19\right)$, which is opposite to our prediction of $H 3$.

$$
\text { Food regime (indexed by mean chlorophyll-a) showed a negative relationship with egg }
$$
size, in agreement with our prediction of hypothesis $H 2\left(\beta_{C h l}=-0.27 ; 95 \%\right.$ CI: $-0.37--0.16$; Fig. 2b). Increasing mean chlorophyll-a from 0.1 to $1 \mathrm{mg} \mathrm{m}^{-3}$ was associated with a c. 1.3fold decrease in egg size after holding all the remaining variables constant. Chlorophyll-a seasonality had a stronger effect than that observed for mean chlorophyll-a $\left(\beta_{\text {SeasonChl }}=-0.69\right.$; 95\% CI: $-0.87--0.51$ ), in agreement with our prediction of $H 3$ : higher seasonality was associated with smaller egg sizes. Additionally, increasing the colour of chlorophyll-a noise (i.e. more temporally-autocorrelated food regime) was associated with a larger egg size ( $\beta_{\theta C h l}$ $8=0.27$; 95\% CI: $0.14-0.40$ ), also in line with our prediction of $H 3$ (Fig. 3). 
300

Here we present a formal and comprehensive test of the role of the environment in shaping marine fish egg size at a global scale, after controlling for potential effects that are attributable to species and phylogenetic relatedness. Importantly, our dataset is composed of geo-located data only, instead of averages for species, thus it is well suited to evaluate the direct effects of the environment on egg size. Our approach builds on decades of empirical (Rass, 1941; Thorson, 1950; Marshall, 1953; Kasimatis \& Riginos, 2016), experimental (Kokita, 2003; Shama, 2015), theoretical (Smith \& Fretwell, 1974; Parker \& Begon, 1986) and conceptual work (Winemiller \& Rose, 1992) documenting the effects of the environment on egg size. On the one hand, our parsimonious model largely confirms predictions based on previous findings, indicating that higher temperatures are associated with smaller egg sizes (Fig. 2a; Rass, 1941; Marshall, 1953; Kokita, 2003; Kasimatis \& Riginos, 2016). On the other hand, for the first time, our results support previously untested concepts relating the effects of environmental predictability (here measured as seasonality and the colour of environmental noise) on marine fish egg size (Winemiller \& Rose, 1992).

Higher mean temperatures were home to fish with smaller egg size, largely supporting Rass's rule (Rass, 1941), and hence our results add to the vast literature reporting the effects of temperature on fish egg size (Rass, 1941; Marshall, 1953; Kokita, 2003; Laptikhovsky, 2006; Shama, 2015; Kasimatis \& Riginos, 2016) and effects of temperature on egg size in aquatic organisms more generally (Marshall et al., 2012). Environments that are seasonal with respect to temperature were associated with a larger egg size, but we note we cannot disentangle this effect from those of mean temperature because these two variables were substantially correlated. Importantly, temperature is predicted to have multiple effects, within and among species. Within species, for example, increasing temperatures are associated with an earlier onset of maturity and declines in maximum adult body size (i.e. temperature-size rule; Zuo et al., 2012). Considering that, within a population, smaller females may produce smaller eggs (e.g. Braga Goncalves et al., 2011), increasing ocean warming will likely decrease the reproductive contribution of populations via effects on both female and egg size (Kokita, 2003). Moreover, warming oceans are expected to alter current distributions of some tropical and temperate fish species (e.g. Feary et al., 2014). Thus, changes in temperature will likely 
change the dynamics and quality of larvae provided in the system by altering their richness, abundance, size, and dispersal capacity (Rass, 1941; Marshall, 1953; Kokita, 2003; O'Connor et al., 2007; Hixon et al., 2014).

Our results highlight congruences between latitudinal patterns in fish and marine invertebrate egg size. Particularly, both groups exhibit a general increase in egg size with increasing latitude (Fig. 1; Marshall et al., 2012) despite a substantial variation around this central tendency. A portion of this variation is explained by incorporating the additional moments of distributions of environmental factors. Our results indicate that productivity (here measured as chlorophyll-a as a proxy) mean, seasonality and noise colour influence patterns in marine fish egg size, in agreement with the early propositions of Thorson (1950), which were recently corroborated by Marshall \& Burgess (2015). Interestingly, our results (i.e. directions of effects) are consistent with the recent analysis of environmental predictability on marine invertebrates egg size with feeding larvae (Marshall \& Burgess, 2015). Our findings may therefore reflect fundamental commonalities regarding how feeding larvae of ectotherms (both zoo-planktivorous fish and phyto-planktivorous invertebrates) respond to environmental predictability.

To the best of our knowledge, our study presents the first formal test of the effects of environmental predictability on marine fish egg size at the global scale. Specifically, we discuss our results in light of the three extreme strategies in the trilateral continuum proposed by Winemiller \& Rose (1992). Environments with autocorrelated fluctuations (i.e. reddened noise) with a low mean food regime exhibit eggs that are $86.6 \%$ larger than the average, consistent with the Equilibrium strategy suggested by Winemiller \& Rose (1992). Similarly, our results indicate that environments with high seasonality and low mean food regime will have eggs that are on average $10.5 \%$ smaller, consistent with a Periodic strategy sensu Winemiller \& Rose (1992). However, our results indicate that environments with low seasonality and white-coloured noise (i.e. not temporally autocorrelated) have eggs that are $15.2 \%$ larger on average, contrary to the expectations of Winemiller and Rose's suggested Opportunistic strategy. Instead, this finding is consistent with Einum \& Fleming (2004) suggestion that mothers employ a conservative bet-hedging strategy under such conditions. Einum \& Fleming (2004) suggested that when mothers cannot anticipate the environment of their offspring, 
they should insulate them from poor conditions through better provisioning. Overall our findings strongly support the notion that environmental quality directly affects egg size (Smith \& Fretwell, 1974; Parker \& Begon, 1986; Rollinson et al., 2013; Shama, 2015). Importantly, all three components of environmental variation: mean, seasonality, and colour of environmental noise drove the patterns in egg size.

After accounting for the effects of the environment and phylogeny, a portion of the variation in the data was left unexplained (i.e. residuals in Fig. 2). For the majority of our records, we were not able to properly assess the depth in which the egg was collected. Depth is known to affect patterns in egg size in marine organisms (Laptikhovsky, 2006). So it is possible that some of our records for "tropical" species were obtained at greater depths were temperatures are much lower. Another aspect that could influence part of the unexplained variance is spawning mode (Kasimatis \& Riginos, 2016). However, our phylogenetic hierarchical model already accounts for clade-specific deviations that drive substantial changes in egg size (e.g. brooding in seahorses and cardinalfishes), and adding spawning mode to the model, as tested through our hypothesis $H 4$, did not significantly improve model fit. This could be due to the fact that some spawning modes are under-represented in the database (e.g. brooders and scatterers), and therefore future studies with more estimates for these groups might be able to resolve the parameter estimates with greater precision.

Here we synthesised large amounts of data to uncover the role of multiple environmental components in shaping marine fish egg size. By doing so, we have confirmed previous results reported in the literature as well as revealed novel patterns. Particularly, our analysis formally decomposed two axes of environmental predictability, showing that they both affect egg size, and in ways consistent to those observed in marine invertebrates, suggesting commonalities in how ectotherm egg size responds to environmental change. Future environmental change in the world's oceans will not only drive the average environmental state at different locations, but also their degree of environmental predictability, so our study suggests that anthropogenically-mediated changes in the environment will have profound effects on the distribution of marine life histories. 


\section{Acknowledgments}

We would like to thank M Elgar for kindly providing data from previous compilations. We also thank CR White, C Olito and the users on the rstan-users Google group (A Gelman, B Carpenter, M Espe, P-C Bürkner, P Smits and M Betancourt) for invaluable advice on hierarchical phylogenetic models. Thanks to DR Robertson for information regarding spawning mode. Special thanks to L Chapman for 1 year of full-time dedication helping the first author compile the database, and the Interlibrary Loan Team at Monash University for helping us access older literature.

\section{Statement of authorship}

All authors conceived the study; DRB compiled and standardised the dataset; DRB and SCB analysed the data; DRB wrote the first draft of the manuscript, and all authors contributed substantially to revisions.

\section{Data accessibility statement}

All data, R packages, and code (data manipulation, analyses, figures and tables) can be downloaded from our GitHub repositories (www.github.com/dbarneche/noaaErddap; www.github.com/dbarneche/envPred; https://github.com/dbarneche/fishEggSize).

\section{Biosketches}

Diego Barneche studies theoretical and empirical approaches that aim to unify evolutionary ecology across levels of biological organisation. He is passionate about data, code, and reproducible science.

Scott Burgess studies life history evolution, dispersal, and population dynamics of coastal marine species using experimental and modelling approaches.

Dustin Marshall works on marine life histories and is increasingly interested in mechanistic explanations for the diversity of life histories found in the sea. 


\section{References}

412 Betancur-R., R., Broughton, R.E., Wiley, E.O., Carpenter, K., López, J.A., Li, C., Holcroft,

413 N.I., Arcila, D., Sanciangco, M., Cureton II, J.C., Zhang, F., Buser, T., Campbell, M.A., 414 Ballesteros, J.A., Roa-Varon, A., Willis, S., Borden, W.C., Rowley, T., Reneau, P.C., Hough, 415 D.J., Lu, G., Grande, T., Arratia, G. \& Ortí, G. (2013) The tree of life and a new classification 416 of bony fishes. PLOS Currents Tree of Life, 1.

417 Braga Goncalves, I., Ahnesjö, I. \& Kvarnemo, C. (2011) The relationship between female 418 body size and egg size in pipefishes. Journal of Fish Biology, 78, 1847-1854.

419 Brown, J.H. (1973) Species diversity of seed-eating desert rodents in sand dune habitats.

420 Ecology, 54, 775-787.

421 Burgess, S.C. \& Marshall, D.J. (2014) Adaptive parental effects: The importance of estimat422 ing environmental predictability and offspring fitness appropriately. Oikos, 123, 769-776.

423 Bürkner, P. (2017) brms: An R package for Bayesian multilevel models using Stan. Journal 424 of Statistical Software, Articles, 80, 1-28.

425 Cohen, D. (1966) Optimizing reproduction in a randomly varying environment. Journal of 426 Theoretical Biology, 12, 119-129.

427 Colwell, R.K. (1974) Predictability, constancy, and contingency of periodic phenomena. 428 Ecology, 55, 1148-1153.

429 Crump, M.L. (1981) Variation in propagule size as a function of environmental uncertainty 430 for tree frogs. The American Naturalist, 117, 724-737.

431 Dormann, C.F., Elith, J., Bacher, S., Buchmann, C., Carl, G., Carré, G., Marquéz, J.R.G., 432 Gruber, B., Lafourcade, B., Leitão, P.J., Münkemüller, T., McClean, C., Osborne, P.E., 433 Reineking, B., Schröder, B., Skidmore, A.K., Zurell, D. \& Lautenbach, S. (2013) Collinearity: 434 A review of methods to deal with it and a simulation study evaluating their performance. 435 Ecography, 36, 27-46.

436 Duarte, C.M. \& Alcaraz, M. (1989) To produce many small or few large eggs: A size437 independent reproductive tactic of fish. Oecologia, 80, 401-404. 
438 Einum, S. \& Fleming, I.A. (2002) Does within $\square$ population variation in fish egg size reflect 439 maternal influences on optimal values? The American Naturalist, 160, 756-765.

440 Einum, S. \& Fleming, I.A. (2004) Environmental unpredictability and offspring size: Conser441 vative versus diversified bet-hedging. Evolutionary Ecology Research, 6, 443-455.

442 Elgar, M.A. (1990) Evolutionary compromise between a few large and many small eggs: 443 Comparative evidence in teleost fish. Oikos, 59, 283-287.

444 Feary, D.A., Pratchett, M.S., J Emslie, M., Fowler, A.M., Figueira, W.F., Luiz, O.J., Naka445 mura, Y. \& Booth, D.J. (2014) Latitudinal shifts in coral reef fishes: Why some species do 446 and others do not shift. Fish and Fisheries, 15, 593-615.

447 Frédérich, B., Sorenson, L., Santini, F., Slater, G.J. \& Alfaro, M.E. (2013) Iterative ecological 448 radiation and convergence during the evolutionary history of damselfishes (pomacentridae). 449 The American Naturalist, 181, 94-113.

450 Fritzsche, R.A. (1978) Development of fishes of the mid-atlantic bight: An atlas of egg, larval 451 and juveniles stages, Fish; Wildlife Service U.S. Department of Interior.

452 Fronhofer, E.A., Stelz, J.M., Lutz, E., Poethke, H.J. \& Bonte, D. (2014) Spatially correlated 453 extinctions select for less emigration but larger dispersal distances in the spider mite Tetrany454 chus urticae. Evolution, 68, 1838-1844.

455 Glynn, E.F., Chen, J. \& Mushegian, A.R. (2006) Detecting periodic patterns in unevenly 456 spaced gene expression time series using lomb-Scargle periodograms. Bioinformatics, 22, $457 \quad 310-316$.

458 Grafen, A. (1989) The phylogenetic regression. Philosophical Transactions of the Royal Soci459 ety of London B: Biological Sciences, 326, 119-157.

460 Hadfield, J.D. \& Nakagawa, S. (2010) General quantitative genetic methods for comparative 461 biology: Phylogenies, taxonomies and multi-trait models for continuous and categorical char462 acters. Journal of Evolutionary Biology, 23, 494-508.

463 Halley, J.M. \& Kunin, W.E. (1999) Extinction risk and the 1/f family of noise models. Theo464 retical Population Biology, 56, 215-230. 
465 Hastings, A. (1983) Can spatial variation alone lead to selection for dispersal? Theoretical 466 Population Biology, 24, 244-251.

467 Hixon, M.A., Johnson, D.W. \& Sogard, S.M. (2014) BOFFFFs: On the importance of con468 serving old-growth age structure in fishery populations. ICES Journal of Marine Science, 71, 4692171.

470 Housworth, E.A., Martins, E.P. \& Lynch, M. (2004) The phylogenetic mixed model. The 471 American Naturalist, 163, 84-96.

472 Kasimatis, K. \& Riginos, C. (2016) A phylogenetic analysis of egg size, clutch size, spawn473 ing mode, adult body size, and latitude in reef fishes. Coral Reefs, 35, 387-397.

474 Kokita, T. (2003) Potential latitudinal variation in egg size and number of a geographically 475 widespread reef fish, revealed by common-environment experiments. Marine Biology, 143, $476 \quad 593-601$.

477 Laptikhovsky, V. (2006) Latitudinal and bathymetric trends in egg size variation: A new look 478 at thorson's and rass's rules. Marine Ecology, 27, 7-14.

479 Marshall, D.J. \& Burgess, S.C. (2015) Deconstructing environmental predictability: Season480 ality, environmental colour and the biogeography of marine life histories. Ecology Letters, 18, $481 \quad 174-181$.

482 Marshall, D.J., Krug, P.J., Kupriyanova, E.K., Byrne, M. \& Emlet, R.B. (2012) The biogeog483 raphy of marine invertebrate life histories. Annual Review of Ecology, Evolution, and System484 atics, $\mathbf{4 3}, 97-114$.

485 Marshall, N.B. (1953) Egg size in arctic, antarctic and deep-sea fishes. Evolution, 7, 328486341.

487 Meiri, S. (2011) Bergmann's rule - what's in a name? Global Ecology and Biogeography, 20, $488 \quad 203-207$.

489 Michonneau, F., Brown, J.W. \& Winter, D.J. (2016) Rotl: An r package to interact with the 490 open tree of life data. Methods in Ecology and Evolution, n/a-n/a.

491 Morrongiello, J.R., Bond, N.R., Crook, D.A. \& Wong, B.B.M. (2012) Spatial variation in 492 egg size and egg number reflects trade-offs and bet-hedging in a freshwater fish. Journal of 
517 Slobodkin, L.B. \& Sanders, H.L. (1969) On the contribution of environmental predictability

518 to species diversity. Diversity and stability in ecological systems Brookhaven symposia in

519 biology. (ed. by G.M. Woodwell) and H.H. Smith), Brookhaven National Laboratory, Upton,

520 NY, USA.

Moser, H.G. (1996) The early stages of fishes in the california current region. atlas no. 33, Allen Press, Lawrence, Kansas, USA.

O’Connor, M.I., Bruno, J.F., Gaines, S.D., Halpern, B.S., Lester, S.E., Kinlan, B.P. \& Weiss, J.M. (2007) Temperature control of larval dispersal and the implications for marine ecology, evolution, and conservation. Proceedings of the National Academy of Sciences, 104, 12661271.

Pagel, M. (1999) Inferring the historical patterns of biological evolution. Nature, 401, 877884.

Paradis, E., Claude, J. \& Strimmer, K. (2004) APE: Analyses of phylogenetics and evolution in R language. Bioinformatics, 20, 289-290.

Parker, G.A. \& Begon, M. (1986) Optimal egg size and clutch size: Effects of environment and maternal phenotype. The American Naturalist, 128, 573-592.

Rass, T.S. (1941) Geograficheskie parallelizmy v stroenii kostistykh ryb severnykh morei (Analogous or parallel variations in structure and development of fishes in Northern and Arctic seas), MOIP, Moscow, Russia.

Rollinson, N., Hutchings, J.A., Sherratt, A.E.T.N. \& McPeek, E.M.A. (2013) Environmental quality predicts optimal egg size in the wild. The American Naturalist, 182, 76-90.

Rubio de Casas, R., Willis, C.G., Pearse, W.D., Baskin, C.C., Baskin, J.M. \& CavenderBares, J. (2017) Global biogeography of seed dormancy is determined by seasonality and seed size: A case study in the legumes. New Phytologist, 214, 1527-1536.

Shama, L.N.S. (2015) Bet hedging in a warming ocean: Predictability of maternal environment shapes offspring size variation in marine sticklebacks. Global Change Biology, 21, $4387-4400$. 
521 Smith, C.C. \& Fretwell, S.D. (1974) The optimal balance between size and number of off522 spring. The American Naturalist, 108, 499-506.

523 Thorson, G. (1950) Reproductive and larval ecology of marine bottom invertebrates. Biologi524 cal Reviews, 25, 1-45.

525 Travis, J.M.J. (2001) The color of noise and the evolution of dispersal. Ecological Research, $52616,157-163$.

527 Vasseur, D.A. \& Yodzis, P. (2004) The color of environmental noise. Ecology, 85, 11465281152.

529 Vasseur, D.A., DeLong, J.P., Gilbert, B., Greig, H.S., Harley, C.D.G., McCann, K.S., Savage, 530 V., Tunney, T.D. \& O’Connor, M.I. (2014) Increased temperature variation poses a greater risk 531 to species than climate warming. Proceedings of the Royal Society of London B: Biological 532 Sciences, 281.

533 Vehtari, A., Gelman, A. \& Gabry, J. (2016) loo: Efficient leave-one-out cross-validation and 534 WAIC for Bayesian models.

535 Winemiller, K.O. \& Rose, K.A. (1992) Patterns of life-history diversification in North Ameri536 can fishes: Implications for population regulation. Canadian Journal of Fisheries and Aquatic 537 Sciences, 49, 2196-2218.

538 Zuo, W., Moses, M.E., West, G.B., Hou, C. \& Brown, J.H. (2012) A general model for effects 539 of temperature on ectotherm ontogenetic growth and development. Proceedings of the Royal 540 Society of London B: Biological Sciences, 279, 1840-1846. 
542 Table 1. Pearson correlation ( $n=1078$ observations) among predictor environmental variables 543 after excluding data with environmental coverage $<87 \%$ (see methods). Bold values represent 544 statistically significant correlations (i.e. $p<0.001$ ).

\begin{tabular}{llrrrrrr} 
& & $\begin{array}{r}\text { SST } \\
\text { (Season.) }\end{array}$ & $\begin{array}{r}\text { SST } \\
\text { (Colour) }\end{array}$ & $\begin{array}{r}\text { SST } \\
\text { (Mean) }\end{array}$ & $\begin{array}{r}\text { Chl } \\
\text { (Season.) }\end{array}$ & $\begin{array}{r}\text { Chl } \\
\text { (Colour) }\end{array}$ & Chl (Mean) \\
\cline { 2 - 7 } 545 & SST (Season.) & - & $\mathbf{0 . 6 8}$ & $\mathbf{- 0 . 7 3}$ & $\mathbf{0 . 3 9}$ & $\mathbf{- 0 . 2 2}$ & $\mathbf{0 . 2 7}$ \\
SST (Colour) & $\mathbf{0 . 6 8}$ & - & $\mathbf{- 0 . 7 1}$ & $\mathbf{0 . 2 4}$ & -0.05 & $\mathbf{0 . 3 9}$ \\
& SST (Mean) & $\mathbf{- 0 . 7 3}$ & $\mathbf{- 0 . 7 1}$ & - & $-\mathbf{0 . 3 2}$ & -0.03 & $\mathbf{- 0 . 4 9}$ \\
& Chl (Season.) & $\mathbf{0 . 3 9}$ & $\mathbf{0 . 2 4}$ & $\mathbf{- 0 . 3 2}$ & - & $\mathbf{0 . 1 6}$ & $\mathbf{- 0 . 1 6}$ \\
Chl (Colour) & $\mathbf{- 0 . 2 2}$ & -0.05 & -0.03 & $\mathbf{0 . 1 6}$ & - & -0.02 \\
546 & Chl (Mean) & $\mathbf{0 . 2 7}$ & $\mathbf{0 . 3 9}$ & $\mathbf{- 0 . 4 9}$ & $\mathbf{- 0 . 1 6}$ & -0.02 & - \\
\cline { 2 - 7 }
\end{tabular}

547 Table 2. Average estimates and 95\% posterior credible intervals (of Bayesian posterior distri-

548 butions) for fitted parameters in the parsimonious model.

\begin{tabular}{lrrrr}
\hline Parameter & $\begin{array}{r}\text { Mean } \\
\text { Estimate }\end{array}$ & $\mathbf{2 . 5 \%}$ & $\mathbf{9 7 . 5 \%}$ & $\begin{array}{r}\text { Effective } \\
\text { sampling }\end{array}$ \\
\hline Random Effects & & & & \\
$\sigma_{\gamma \text { spp }}$ & 0.24 & 0.20 & 0.28 & 5346 \\
$\sigma_{\gamma p h y}$ & 1.57 & 1.13 & 2.04 & 4275 \\
& & & & \\
Fixed effects & & & & \\
$\beta_{0}$ (intercept) & 0.43 & -1.31 & 2.24 & 6309 \\
$\beta_{\text {SST }}$ & -0.02 & -0.03 & -0.01 & 17046 \\
$\beta_{\text {Chl }}$ & -0.27 & -0.37 & -0.16 & 22500 \\
$\beta_{\theta C h l}$ & 0.27 & 0.14 & 0.40 & 22500 \\
$\beta_{\text {SeasonChl }}$ & -0.69 & -0.87 & -0.51 & 22500 \\
$\beta_{\text {SeasonSST }}$ & 0.82 & 0.47 & 1.19 & 12568 \\
\hline
\end{tabular}


551 Figure 1. Raw data showing the relationship between egg size (diameter in $\mathrm{mm}$ ) and abso-

552 lute latitude. Blue and red points represent data from the southern and northern hemispheres

553 respectively.

554 Figure 2. Fish egg size (diameter in $\mathrm{mm}$ ) as a function of (a) mean SST (b) and mean

555 chlorophyll-a. Parameter estimates (parsimonious model in Table 2) were obtained using

556 Bayesian phylogenetic hierarchical models. The effects of environmental predictability and

557 mean chlorophyll-a on egg size were controlled for in $(a)$, and the environmental predictability

558 and mean SST in (b). Random effects attributable to relatedness among species and species

559 uniqueness ( $\gamma p h y$ and $\gamma s p p$ in eqn 1) have also been controlled for. Thus, the deviations from

560 the mean model fit represent the actual model residuals. Top-left equations show average

561 fixed-effect predictions. Dashed thick line represents average posterior fixed-effect fit, and

562 dashed thin lines represent the $95 \%$ posterior credible intervals extracted from 22,500 MCMC

563 samples from our Bayesian analysis.

564 Figure 3. Predictive surface between chlorophyll-a seasonality and noise colour on the aver-

565 age size of marine fish eggs (diameter in $\mathrm{mm}$ ). Each black circle represents a separate obser-

566 vation in our dataset and the colour coding represents the predicted surface using parameters

567 from our parsimonious Bayesian phylogenetic hierarchical model (Table 2). Predicted egg

568 sizes were calculated for mean SST and chlorophyll-a, as well as mean SST seasonality. 